\title{
PROKLA-Redaktion
}

\section{Die USA vor, mit und nach Trump}

D ie Rede war von einem »Scherbenhaufen «, den der scheidende Präsident hinterlassen hatte, »die weitere, deutliche Verschärfung der Einkommens- und Vermögensungleichheit im Land mit den Mitteln der Steuer- und Sozialpolitik; die Lizenz zum Plündern von natürlichen Ressourcen in ausgewiesenen Naturschutzgebieten und der Widerstand gegen jede internationale Politik des Klimaschutzes - die Liste lässt sich mühelos fortsetzen. Wie die Umfragen wenige Wochen vor der Wahl anzeigen, hätte all dies allerdings möglicherweise noch immer nicht ausgereicht, die Republikaner aus dem Weißen Haus zu treiben.« Eine treffende Beschreibung der Lage in den USA im Herbst 2020? Nein, so stand es im Editorial der PROKLA 153 aus dem Jahr 2008, einem Heft, das vor rund zwölf Jahren unter dem Titel Die USA nach Bush erschien. Wenngleich auch dieser Präsident gewisse Verhaltensauffälligkeiten an den Tag legte, ging es uns damals weniger um dessen Person als um die strukturellen Hintergründe für den katastrophalen Einbruch der Finanzmärkte, der die größten Investmentbanken des Landes ins Straucheln brachte, Millionen einfacher Menschen ihre Häuser wegnahm und ihnen nichts als einen Schuldenberg beließ, sowie den Glauben an das segensreiche Wirken freier
Märkte krachend zusammenbrechen ließ - zumindest für eine gewisse Zeit. Wir fragten daher unter anderem nach der Macht des Finanzsektors, nach der vorherrschenden Armutspolitik oder nach der Bedeutung der Evangelikalen - gesellschaftlichen Phänomenen, die mit dem neoliberalen Aufschwung seit Anfang der 1980er-Jahre einhergingen.

Der Scherbenhaufen sah im November 2020 , als Donald Trump zur Wiederwahl antrat, ähnlich aus, er war aber keineswegs der erste seiner Art, wie eine Fixierung auf die exzentrische Person des Präsidenten es häufig nahelegt. In beiden Fällen hatte eine konservative Regierung eine beinharte neoliberale Politik betrieben, doch die gesellschaftliche Krise spitzte sich aus je unterschiedlichen Gründen zu. Damals geschah dies dank der internen Dynamik des Wirtschaftssystems und insbesondere der Finanzmärkte, diesmal durch ein Ereignis, das zwar ebenfalls mit der bedenkenlosen Ressourcennutzung einer auf Wachstum gepolten Wirtschaftsordnung zu tun hat, das Land aber dennoch von außen traf: die Covid-19-Pandemie, die sich in den USA besonders verheerend ausbreitete und auswirkte. Allein bis zum April 2021 führte sie zu mehr als einer halben Million Toten, sodass hier durch die Seuche bis dahin mehr Menschen ums Leben kamen als in je- 
dem anderen Land der Erde. Das ging zum einen darauf zurück, dass Trump gegenüber dem grassierenden Virus das praktizierte, was Benjamin Kunkel eine "sadistische Nonchalance " nannte, also eine Politik betrieb, die sich - wie bei ihm üblich - durch tägliche konfuse und hetzerische Twitter-Meldungen, eine haarsträubende Ignoranz von Fakten, unzählige Lügereien und bizarre Auftritte in der Öffentlichkeit auszeichnete. Zum anderen kamen dabei die notorischen Defizite des Gesundheitssystems im reichsten Land der Welt zum Tragen, das vor allem seinen afroamerikanischen und lateinamerikanischen Bürgern und Bürgerinnen oft nicht einmal den elementarsten Schutz gegen die Seuche gewähren konnte.

Auch bei den gesellschaftlichen Folgen zeigten sich keineswegs neue Muster, sondern meist nur eine Verschärfung der seit Jahrzehnten bekannten Verwerfungen: Die Reichen wurden (wieder einmal) noch reicher, die Armen standen Schlange vor kirchlichen oder karitativen Einrichtungen, die kostenlos warmes Essen verteilen. In einem Land, das jahraus, jahrein einen riesigen Überschuss an Lebensmitteln produziert, ist das Problem des Hungers abermals in einer Schärfe an den Tag getreten, wie man das sonst aus Ländern mit dem niedrigsten weltweiten Pro-Kopf-Einkommen kennt. Ein Fünftel der Bevölkerung hat seit dem wirtschaftlichen Einbruch durch die Pandemie nicht immer genug zu essen, ein Achtel muss Lebensmittelgutscheine in Anspruch nehmen. Dazu kommt, dass viele Millionen Menschen auf der Straße, in Zelten oder in Wohnwagen leben, weil sie sich keine Wohnung mehr leisten können. Die Zahl und Größe der Trailer Parks ist in einem Ausmaß angewachsen, das sie sogar für Investmentfirmen interessant werden ließ. Sie haben sie in vielen Fällen den bisherigen kleinen privaten Eigentümern abgekauft, um an ihnen nach ihrer üblichen Gewinnlogik zu verdienen - stets zum Nachteil der dortigen Bewohner und Bewohnerinnen.

Angesichts des wirtschaftlichen und sozialen Elends, das mit der Ausbreitung der Pandemie unübersehbar wurde, griff die Trump-Regierung im Frühjahr 2020 zu Stützungsmaßnahmen, bei denen massenhaft Schecks verteilt wurden, die mit der persönlichen Unterschrift des Präsidenten verziert waren. Parallel dazu wuchsen die Einkommen von Millionären und insbesondere von Milliardären weiter kräftig an, und vor allem Handelsplattformen und IT-Konzerne wiesen Kurssprünge und Gewinne wie nie zuvor auf. Das wirft die Frage auf, wie die Parteien der Republikaner und der Demokraten sich diesen Entwicklungen gegenüber positionierten. Wie kam es, dass die Wahl vom November 2020 keineswegs $\mathrm{zu}$ dem von vielen erwarteten Erdrutschsieg der Demokraten führte? Zwar gewann Joe Biden mit mehr als sieben Millionen Stimmen Vorsprung den popular vote, doch diese Überlegenheit übersetzte sich dank des anachronistischen WahlmännerSystems nur sehr viel weniger in deren Stimmen. Vor allem aber verloren die Demokraten im Repräsentantenhaus mehrere Sitze und haben dort nur noch eine schwache Mehrheit; im Senat ist ein Patt von 50:50 entstanden, sodass sie lediglich mit der Stimme der Vizepräsidentin zu einer Mehrheit kommen. 
Mike Davis untersucht in seinen Anmerkungen zu den November-Wahlen, wie es bei einer ungewöhnlich hohen Wahlbeteiligung vor allem den Republikanern gelungen ist, ihre Wählerschaft mehr als zuvor zu mobilisieren, und identifiziert dabei als entscheidende Gruppen die BewohnerInnen in den Counties des sogenannten Rostgürtels sowie jene im kleinstädtischen Mittleren Westen, in den Vorstädten und im texanischen Grenzland. Für diese Gruppen stand das Thema Wirtschaft im Mittelpunkt und sie meinten, diese würde sich mit den Republikanern besonders gut entwickeln. Margit Mayer analysiert die Politik der Demokraten sowie ihr Verhältnis zur Linken und stellt spiegelbildlich zu den Befunden von Davis fest, dass sich die Demokraten in ihrem Wahlkampf auf die gebildeten metropolitanen Mittelschichten konzentriert und die kleinstädischen sowie die ländlichen Regionen vernachlässigt haben. Gerade dort gelang es anfangs Bernie Sanders, dem führenden Vertreter der progressiven Demokraten, entscheidende Erfolge zu erzielen, doch wurde er in der Folge - wie generell die VertreterInnen dieser Richtung - durch das Partei-Establishment massiv ausgebremst. Es bleibt abzuwarten, wie sich das Verhältnis der verschiedenen Flügel weiter entwickeln wird.

Ungeachtet der persönlichen Misserfolgsgeschichte Trumps - einem wiederholten Bankrotteur, dem nach seiner Amtszeit zahlreiche Prozesse wegen Steuer- und Versicherungsbetrug sowie Bilanzfälschungen drohen - wird ihm von der republikanischen Basis Kompetenz in Angelegenheiten der Wirtschaft zugesprochen. Damit stellt sich die Frage, wie sich die Wirtschaft in den Jahren seiner Amtszeit tatsächlich entwickelt hat. Diesem Thema widmet sich Trevor Evans, der zeigt, dass sich seit den 1980erJahren die wichtigsten makroökonomischen Trends (bei starken zyklischen Schwankungen) unter Trump fortgesetzt haben, etwa die Abschwächung der Wachstumsraten des Sozialprodukts oder der Arbeitsproduktivität. Ebenso wenig neu war in der Zeit seiner Präsidentschaft, dass Unternehmen eine stetig sinkende Neigung zeigten, Investitionen vorzunehmen, sondern lieber dem Druck der Aktionäre nachgaben, ihnen immer höhere Dividenden auszuzahlen oder Aktien von ihnen zurückzukaufen. Abgesehen von dem kurzen Strohfeuer des Anstiegs der Investitionen nach der großen Steuerreform von 2018 und von den Maßnahmen der Deregulierung für den Finanzsektor hat Trump die längerfristigen Entwicklungen somit nur relativ wenig beeinflusst. Der gewaltige wirtschaftliche Einbruch als Folge der Pandemie zwang die Regierung dazu, den bisher hochgehaltenen Fiskalkonservatismus, also die Politik der »schwarzen Null«, weitgehend aufzugeben, und die ersten wirtschaftspolitischen Entscheidungen der neuen Biden-Regierung weisen ebenfalls in die Richtung einer »Dämmerung des Neoliberalismus «. Ob sich eine solche tatsächlich ereignen wird, erscheint angesichts der politischen Kräfteverhältnisse jedoch mehr als fraglich. Sie würde nichts weniger als eine massive Erhöhung der Steuern für Vermögende und gut verdienende Unternehmen voraussetzen.

Die Neuorientierung der Wirtschaftspolitik wird vermutlich eine sehr viel weniger radikale Umkehr bedeuten, als vielfach angenommen wird, wie auch 
Christoph Scherrer in seiner Analyse der Außenwirtschaftspolitik zeigt. Denn diese war unter Präsident Trump trotz aller donnernden America-first-Rhetorik noch nie anders als an den Interessen des US-amerikanischen Kapitals orientiert und in der Praxis keineswegs so konsequent protektionistisch, wie dieser für sich in Anspruch nahm. Auf der anderen Seite dürfte Joe Biden eine Reihe der Maßnahmen seines Vorgängers bestehen lassen und faktisch eine Politik anstreben, die eine "Kreuzung von Obama und Trump « darstellen wird.

Auch der Bereich der Kulturpolitik, den Nick Prasse unter die Lupe nimmt, war in den letzten Jahren vor allem durch langfristig etablierte Strukturen geprägt, insbesondere durch das im europäischen Vergleich minimale staatliche Engagement, demgegenüber der Sektor stets auf Kommerzialisierung setzte, und dort, wo diese nicht erfolgreich sein konnte, auf Philantropie angewiesen blieb. Dass die TrumpRegierung staatliche Ausgaben dazu als »überflüssig « und »verschwenderisch « ansah, dürfte niemanden überraschen. Gerade dieser Bereich und die in ihm häufig prekär Beschäftigten wurden daher von der Covid-19-Epidemie besonders stark getroffen, was allerdings zu verstärkten Bemühungen der Kulturschaffenden geführt hat, sich über Netzwerke neu zu organisieren. Ein weiterer Bereich, dem ein eigener Beitrag gewidmet ist, gilt dem Gefängnissystem, das Lukas Egger näher untersucht. Seit den 1970er-Jahren gibt es in den USA eine für ein westliches Land einzigartig hohe Inhaftierungsrate, die zum allergrößten Teil die schwarze Bevölkerung traf und ganz überwiegend auf Drogendelikte zu- rückging. Der Autor verfolgt, inwieweit hier die Dimensionen der Klassenzugehörigkeit und der Hautfarbe miteinander verschränkt sind, und wie sich das System der Masseninhaftierungen unter der Trump-Regierung entwickelt hat.

Auch wenn Trump selbst gerne als Vertreter von Law and Order auftrat, war sein Umgang mit staatlichen Institutionen und Gesetzen stets von persönlichem Opportunismus geprägt, was sich insbesondere in seinem Verhältnis zu rechten Milizen und in seiner Reaktion auf die verlorene Präsidentenwahl zeigte. Milizen wie die Proud Boys, die zunächst gegenüber dem Staat überwiegend kritisch eingestellt waren und seit der neoliberalen Wende einen starken Aufschwung erlebten, erfuhren von ihm Anerkennung und Zuspruch. Carolyn Gallaher und Jaclyn Fox gehen in ihrem Beitrag der Frage nach, ob sich diese Milizen zu Paramilitärs entwickeln würden. Sie sehen dabei in der Vergangenheit zwei mögliche Pfade. Der eine bestand darin, dass die Milizen sich mit den sogenannten Weißen Nationalisten, die einen (weißen) Ethnostaat anstreben, verbündeten; doch standen dem insbesondere diametral entgegengesetzte Haltungen zum Staat entgegen. Der zweite lag darin, dass die unterschiedlichen Gruppierungen einen gemeinsamen Nenner in ihrer Rolle als Erfüllungsgehilfen der Trumpschen Machtansprüche finden würden. Genau das geschah bei dem Angriff auf das Kapitol vom 6. Januar 2021. Sie folgten damit der Aufforderung des abgewählten Präsidenten, sich für ihn, dem man die Wahl angeblich "gestohlen « hatte, stark zu machen. Trump selbst setzte sich nicht, wie viele der bewaffneten 
Randalierenden es erwartet hatten, an die Spitze dessen, was ihnen als "Sturm" auf das Kapitol galt, sondern sah sich die Vorgänge in aller Ruhe zu Hause im Fernsehen an. Das enttäuschte viele, dennoch scheint dies die Faszination, die er auch außerhalb der eigentlichen Miliz-Angehörigen immer noch ausübt, nicht wirklich gebrochen zu haben. Obwohl es inzwischen Belege dafür gibt, dass Gelder, die in den letzten Monaten für die Kampagne seiner Wiederwahl eingesammelt wurden, zu einem groBen Teil veruntreut wurden, hielt der Zustrom des Geldes von KleinspenderInnen, die ihm ihre unverbrüchliche Treue bezeugen wollen, weiter an. Die anhaltende Bedeutung dieser grassrootsBewegung belegt, wie tief gespalten die Gesellschaft trotz aller Appelle der neuen Regierung zur Versöhnung bleibt.
Neben diesen Beiträgen widmet sich auch der "Einspruch" von Mitchell G. Ash den Zuständen in den USA. Er stellt sich die Frage, ob die Ursache für die aktuelle politische Krise des Landes in deren Verfassung von 1787 liegt. Doch die Verfassung ist ursprünglich in keiner Weise »demokratisch « und wird bis heute sehr unterschiedlich interpretiert - so meinten etwa viele von denen, die ins Kapitol eindrangen, sie würden nichts anderes tun, als sie zu verteidigen.

Jenseits des Schwerpunkts erscheint ein Text von Stefanie Hürtgen, die darin ihre Kritik an dem Konzept der »imperialen Lebensweise«, wie es von Ulrich Brand und Markus Wissen in ihrem gleichnamigen Buch vertreten wird, fortsetzt. Der erste Teil erschien in $\mathrm{H}$. 198/2020.

\section{Der PROKLA Förderverein}

Die PROKLA erscheint seit 1971 und bietet politisch engagierte sozialwissenschaftliche und ökonomische Analysen. Allein von den Verkaufserlösen kann sich die PROKLA nicht finanzieren, und in die Abhängigkeit von Parteien oder großen Verlagen wollte sie sich nie begeben. Deshalb wird die PROKLA von einem Förderverein herausgegeben, der »Vereinigung zur Kritik der politischen Ökonomie e.V.«, die jährlich in ihrer Vollversammlung die Redaktion der Zeitschrift wählt und die nächsten Themenschwerpunkte diskutiert.

Kritische Sozialwissenschaft kann nicht dem Markt überlassen werden. Ohne solidarische Strukturen und finanzielle Unterstützung sind Zeitschriften wie die PROKLA kaum möglich. Die finanziellen Beiträge der Vereinsmitglieder ermöglichen das Erscheinen der PROKLA, sie schaffen die Voraussetzungen für Kontinuität und Planbarkeit, wie sie für die Redaktionsarbeit unabdingbar sind. Wir freuen uns über weitere Mitglieder, regelmäßige Spenden oder einmalige Zuwendungen. Weitere Informationen teilen wir gerne per E-Mail mit (redaktion@prokla.de).

Vereinigung zur Kritik der politischen Ökonomie e.V. | Postbank Berlin | IBAN: DE17 100100100538135100 | BIC: PBNKDEFF 